\title{
Some non-trivial PL knots whose complements are homotopy circles
}

by

\author{
Greg Friedman (Nashville, TN)
}

Dedicated to the memory of Jerry Levine (May 4, 1937 - April 8, 2006)

\begin{abstract}
We show that there exist non-trivial piecewise linear (PL) knots with isolated singularities $S^{n-2} \subset S^{n}, n \geq 5$, whose complements have the homotopy type of a circle. This is in contrast to the case of smooth, PL locally flat, and topological locally flat knots, for which it is known that if the complement has the homotopy type of a circle, then the knot is trivial.
\end{abstract}

It is well known that if the complement of a smooth, piecewise linear (PL) locally flat, or topological locally flat knot $K \subset S^{n}, K \cong S^{n-2}, n \geq 5$, has the homotopy type of a circle, then $K$ is equivalent to the standard unknot in the appropriate category (see Stallings [11] for the topological case and Levine [6] and $[8, \S 23]$ for the smooth and PL cases). This is also true of classical knots $S^{1} \hookrightarrow S^{3}$ (see $[10, \S 4$.B]), for which these categories are all equivalent, and in the topological category for locally flat knots $S^{2} \hookrightarrow S^{4}$ by Freedman [2, Theorem 6].

By contrast, Freedman and Quinn showed in $[3, \S 11.7]$ that any classical knot with Alexander polynomial 1 bounds a topological locally flat $D^{2}$ in $D^{4}$ whose complement is a homotopy circle, and by collapsing the boundary, one obtains a singular $S^{2}$ in $S^{4}$ with the same property. In the same dimensions, Boersema and Taylor [1] constructed a specific example of a PL knot with an isolated singularity whose complement is a homotopy circle. It follows by taking iterated suspensions that there are non-trivial PL embeddings $S^{n-2} \hookrightarrow S^{n}$ in all dimensions $n \geq 4$ whose complements are homotopy circles, though this process will lead to increasingly more complicated singularities. In this note, we construct PL knots for any $n \geq 5$ that are locally flat except at one point and whose complements are homotopy circles.

2000 Mathematics Subject Classification: Primary 57Q45; Secondary 55P10.

Key words and phrases: knot theory, PL knot, simple knot, knot complement. 
To construct the knots with the desired properties, it will suffice to construct for each $n \geq 5$ a PL locally flat disk knot $L \subset D^{n}$ such that $D^{n}-L \sim_{\text {h.e. }} S^{1}$ and such that the PL locally flat boundary sphere knot $\partial L \subset \partial D^{n}$ is non-trivial. By a PL locally flat disk knot $L \subset D^{n}$, we mean the image of a PL locally flat embedding $D^{n-2} \hookrightarrow D^{n}$ such that $\partial L \subset \partial D^{n}$ is a locally flat sphere knot and $\operatorname{int}(L) \subset \operatorname{int}\left(D^{n}\right)$. This will suffice since, if such a disk knot exists, we may then adjoin the cone on the boundary pair $\left(\partial D^{n}, \partial L\right)$ to obtain a PL sphere knot $K \subset S^{n}$ that is locally flat except at the cone point:

$$
\begin{array}{ccccc}
K & = & L & \cup_{\partial L} & c(\partial L) \\
\cap & \cap & & \cap \\
S^{n} & = & D^{n} & \cup_{\partial D^{n}} & c\left(\partial D^{n}\right)
\end{array}
$$

It is clear that $S^{n}-K \sim_{\text {h.e. }} D^{n}-L$, so if the complement of $L$ is a homotopy circle then so will be that of $K$. Furthermore, $K$ will be non-trivial since the link pair of the cone point will be non-trivially knotted, which is impossible in the unknot, which is locally flat.

So we construct such a disk knot. The procedure will be based upon that given by the author in [4] for constructing certain Alexander polynomials of disk knots, which in turn was a generalization of Levine's construction of sphere knots with given Alexander polynomials in [7]. All spaces and maps will be in the PL category without further explicit mention.

Suppose that $n \geq 5$, and let $U$ be the trivial disk knot $U \subset D^{n}$, i.e. $D^{n}$ may be identified with the unit ball in $\mathbb{R}^{n}$ such that $U$ is the intersection of $D^{n}$ with the coordinate plane $\mathbb{R}^{n-2} \subset \mathbb{R}^{n}$. We can assume that $U$ bounds an embedded $(n-1)$-disk $V$ in $D^{n}$, that $\partial U$ bounds an $(n-2)$ disk $F$ in $\partial D^{n}$, that $\partial V=U \cup F$, and that $\operatorname{int}(V) \subset \operatorname{int}\left(D^{n}\right)$. Embed an unknotted $S^{n-3}$ into $\partial D^{n}=S^{n-1}$ so that it is not linked with $\partial U$ and does not intersect $F$ (in fact, we may assume that the new $S^{n-3}$ and $F$ are in opposite hemispheres of $\left.\partial D^{n}\right)$. We use the standard framing of the new unknotted $S^{n-3}$ to attach an $(n-2)$-handle to $D^{n}$, obtaining a space homeomorphic to $S^{n-2} \times D^{2}$ and containing $V$ in a trivial neighborhood of some point on the boundary. Let $C_{0}=S^{n-2} \times D^{2}-U$. Since $\pi_{1}\left(D^{n}-U\right) \cong \mathbb{Z}, \pi_{1}\left(C_{0}\right) \cong \mathbb{Z}$ by the Seifert-van Kampen theorem. Let $\widetilde{C}_{0}$ be the infinite cyclic cover of $C_{0}$ associated with the kernel of the homomorphism $\pi_{1}\left(C_{0}\right)=\mathbb{Z} \rightarrow \mathbb{Z}$ determined by linking number with $U$. Let $X_{0}=\partial\left(S^{n-2} \times D^{2}\right)-\partial U$, and let $\widetilde{X}_{0}$ be the infinite cyclic cover of $X_{0}$ in $\widetilde{C}_{0}$.

As in the usual construction of infinite cyclic covers in knot theory (see, e.g., Rolfsen [10]), we can form $\widetilde{C}_{0}$ by a cut and paste procedure: we cut $C_{0}$ along $V$ to obtain $Y_{0}$ and then glue a countably infinite number of copies of $Y_{0}$ together along the copies of $V$. Since $C_{0}-V \sim_{\text {h.e. }} S^{n-2}$, we have 
$\widetilde{H}_{n-2}\left(\widetilde{C}_{0}\right)=\mathbb{Z}[\mathbb{Z}]=\mathbb{Z}\left[t, t^{-1}\right]$ (where $t$ represents a generator of the group of covering translations) and all other reduced homology groups are trivial. Similarly, since $\partial\left(S^{n-2} \times D^{2}\right)-F$ is a punctured $S^{n-2} \times S^{1}, \widetilde{H}_{*}\left(\widetilde{X}_{0}\right)$ is $\mathbb{Z}[\mathbb{Z}]$ in dimensions $n-2$ and 1 , and trivial otherwise.

It is also apparent that $\pi_{*}\left(\widetilde{C}_{0}\right)$ is trivial for $*<n-2$, while $\pi_{1}\left(\widetilde{X}_{0}\right)$ is free on a countably infinite number of generators. Thus, since $n \geq 5$, $\pi_{2}\left(\widetilde{C}_{0}, \widetilde{X}_{0}\right)$ is also free on a countably infinite number of generators. Meanwhile, for $X_{0}$ itself, $\pi_{1}\left(X_{0}\right)$ is the free group on two generators: one generator corresponds to the generator of $\pi_{1}\left(\partial\left(S^{n-2} \times D^{2}\right)\right)=\pi_{1}\left(S^{n-2} \times S^{1}\right)$ $=\mathbb{Z}$ and the other corresponds to the meridian of the unknotted $\partial U$ (this can be demonstrated by an easy Seifert-van Kampen argument, by considering $\partial U$ to lie in a ball neighborhood of some point). Let $a$ represent the generator corresponding to the meridian of $\partial U$, and let $b$ represent the other described generator. We note that the generator of $\pi_{1}\left(C_{0}\right) \cong \mathbb{Z}$ is also given by $a$, while $b$ is contractible in this larger space.

Consider now the element $\gamma$ of $\pi_{1}\left(X_{0}\right)$ given by $b^{2} a b a^{-1} b^{-1} a b^{-1} a^{-1}$. Since $b=1$ in $\pi_{1}\left(C_{0}\right)$ and $a$ occurs with total exponent 0 in $\gamma$, the image of $\gamma$ in $\pi_{1}\left(C_{0}\right)$ is trivial, so any representative of $\gamma$ is the boundary of a 2-disk $\Gamma$ in $C_{0}$. Since $n \geq 5$, we can assume that $\Gamma$ is properly embedded (see [5, Corollary 8.2.1]). Furthermore, $\gamma$ can be lifted to a closed curve in $\widetilde{X}_{0}$; if we let $c_{i}$ represent the generators of $\pi_{1}\left(\widetilde{X}_{0}\right)$, then any lift of $a$ is a path between adjoining lifts of $X_{0}$ in the cut and paste construction, and $\gamma$ lifts to $\widetilde{\gamma}=c_{0}^{2} c_{1} c_{0}^{-1} c_{1}^{-1} \in \pi_{1}\left(\widetilde{X}_{0}\right)$. In the abelianization $H_{1}\left(\widetilde{X}_{0}\right)$, the image of $\widetilde{\gamma}$ is the same as the image of $c_{0}$, which is a $\mathbb{Z}[\mathbb{Z}]$-module generator of $H_{1}\left(\widetilde{X}_{0}\right)$.

Let $N$ denote an open regular neighborhood of $\Gamma$ in $C_{0}$. We claim that $S^{n-2} \times D^{2}-N$ is homeomorphic to $D^{n}$. In fact, observe that in $S^{n-2} \times S^{1}$, $\gamma$ is homotopic to the standard generator $b=* \times S^{1}$ of $\pi_{1}\left(S^{n-2} \times S^{1}\right)$ (with an appropriate choice of orientations). Thus, in $\left(S^{n-2} \times D^{2}, S^{n-2} \times S^{1}\right)$, the pair $(\Gamma, \gamma)$ is homotopic to the standard generator $* \times D^{2}$ of $\pi_{2}\left(S^{n-2} \times D^{2}\right.$, $\left.S^{n-2} \times S^{1}\right)$. These homotopies can be realized by ambient isotopies by [5, Theorem 10.2]. Then it is clear that $S^{n-2} \times D^{2}-N \cong D^{n-2} \times D^{2} \cong D^{n}$.

Fixing a homeomorphism $S^{n-2} \times D^{2}-N \rightarrow D^{n}$, the image of $U$ is a new disk knot, which we christen $L$. We claim that $L$ is no longer trivial but that its complement is a homotopy circle.

Let $C$ be the complement of an open regular neighborhood of $L$ in $D^{n}$ (the disk knot exterior). Thus $C$ is homotopy equivalent to $D^{n}-L$. Similarly, let $X$ be the exterior of $\partial L$ in $\partial D^{n}=S^{n-1}$. We must study the homotopy and homology of $C, X$, and their coverings.

Lemma $1 . \pi_{1}(C)=\mathbb{Z}$. 
Proof. $C \sim_{\text {h.e. }} D^{n}-L \cong\left(C_{0}-N\right) \cup_{D^{2} \times S^{n-3}} N$. Since $\pi_{1}\left(C_{0}\right) \cong \mathbb{Z}$ and $N \cong D^{n}, \pi_{1}(C) \cong \mathbb{Z}$ by the Seifert-van Kampen theorem.

\section{Lemma 2. $\pi_{1}(X) \cong\left\langle a, b \mid b^{2} a b a^{-1} b^{-1} a b^{-1} a^{-1}\right\rangle$.}

Proof. The effect of the handle subtraction $C_{0}-N$ on the boundary $X_{0}$ is that of a surgery on the embedded curve $\gamma$. Since $\pi_{1}\left(X_{0}\right)$ is free on the generators $a$ and $b$, the result of the surgery is the given group. (Proof: The result of the surgery is $\left(X_{0}-S^{1} \times D^{n-2}\right) \cup D^{2} \times S^{n-3}$, where the $S^{1}$ represents $\gamma$. But since $n \geq 5, \pi_{1}\left(X_{0}-S^{1} \times D^{n-2}\right) \cong \pi_{1}\left(X_{0}\right)$. So by Seifertvan Kampen, $\pi_{1}$ of the result of the surgery is $\pi_{1}\left(X_{0}\right) / \pi_{1}\left(S^{1} \times S^{n-3}\right) \cong$ $\pi_{1}\left(X_{0}\right) / \mathbb{Z}$, where the $\mathbb{Z}$ is generated by $S^{1} \times *$ in $S^{1} \times S^{n-3}$, which is the boundary of the neighborhood of $\gamma$. But any such curve is homotopic to $\gamma$, which represents $b^{2} a b a^{-1} b^{-1} a b^{-1} a^{-1}$.)

Lemma 3. The Alexander modules $\widetilde{H}_{*}(\widetilde{C}), \widetilde{H}_{*}(\widetilde{X})$, and $\widetilde{H}_{*}(\widetilde{C}, \widetilde{X})$ are all trivial.

Proof. Let $\widetilde{\gamma}$ be the lift of $\gamma$ considered above. We can also lift $\Gamma$ to a 2-disk $\widetilde{\Gamma}$ in $\widetilde{C}_{0}$. In fact, we can find a countable number of lifts $\widetilde{\gamma}_{i}$ and $\widetilde{\Gamma}_{i}$, and, since $\Gamma$ is embedded, the $\widetilde{\Gamma}_{i}$ are all disjoint. If $\widetilde{N}_{i}$ then represent the lifts of the regular neighborhood $N, \widetilde{C}_{0}-\coprod_{i} \widetilde{N}_{i}$ will be the infinite cyclic cover of $C_{0}-N \cong D^{n}-L$.

Now consider $\widetilde{X}_{0} \cup \coprod_{i} \widetilde{N}_{i}$. Each intersection $\widetilde{X}_{0} \cap N_{i}$ is homotopy equivalent to a translate of $\widetilde{\gamma}_{i}$, which we know represents the $\mathbb{Z}[\mathbb{Z}]$-module generator of $H_{1}\left(\widetilde{X}_{0}\right)$. It thus follows from the Mayer-Vietoris sequence that $\widetilde{H}_{*}\left(\widetilde{X}_{0} \cup \coprod_{i} \widetilde{N}_{i}\right)$ is trivial except in dimension $n-2$, where it is $\mathbb{Z}[\mathbb{Z}]$. Meanwhile, we already know that $\widetilde{H}_{*}\left(\widetilde{C}_{0}\right)$ is trivial except in dimension $n-2$, where it is also $\mathbb{Z}[\mathbb{Z}]$. Consider the map $H_{n-2}\left(\widetilde{X}_{0} \cup \coprod_{i} \widetilde{N}_{i}\right) \rightarrow H_{*}\left(\widetilde{C}_{0}\right)$. In each module, a $\mathbb{Z}[\mathbb{Z}]$-module generator is represented by a choice of $S^{n-2} \times * \subset S^{n-2} \times S^{1} \subset S^{n-2} \times D^{2}$ that is disjoint from $V$. Thus this homology map is an isomorphism, and it follows that $H_{*}\left(\widetilde{C}_{0}, \widetilde{X}_{0} \cup \coprod_{i} \widetilde{N}_{i}\right)$ is trivial. But by excision, $H_{*}\left(\widetilde{C}_{0}, \widetilde{X}_{0} \cup \coprod_{i} \widetilde{N}_{i}\right) \cong H_{*}(\widetilde{C}, \widetilde{X})$.

Similarly, it follows from easy homological calculations that $\widetilde{H}_{*}(\widetilde{X})$ is trivial. In fact, it can be seen that the construction of $X$ from $X_{0}$ is by a surgery, and upon restriction of our construction to its effect on $X_{0}$, we obtain the construction of Levine for producing smooth sphere knots with given Alexander polynomials in [7]. In this case, the Alexander polynomial is trivial (since $\widetilde{\gamma}$ generates $H_{1}\left(\widetilde{X}_{0}\right)$ ), and it follows from Levine's calculations that $\widetilde{H}_{*}(\widetilde{X})=0$.

Then $\widetilde{H}_{*}(\widetilde{C})$ is also trivial, by the long exact sequence of the pair $(\widetilde{C}, \widetilde{X})$.

Proposition 4. $\pi_{*}\left(D^{n}-L\right) \cong \pi_{*}\left(S^{1}\right)$. 
Proof. By Lemma $1, \pi_{1}(C)=\mathbb{Z}$. Thus the infinite cyclic cover $\widetilde{C}$ is simply connected, and since we also have $\widetilde{H}_{*}(\widetilde{C})=0$ by Lemma 3 , it follows that $\pi_{j}(\widetilde{C})=0$ for all $j>1$ by Hurewicz's theorem. Thus for $j>1$, $\pi_{j}(C)=0$, and $\pi_{*}\left(D^{n}-L\right) \cong \pi_{*}(C) \cong \pi_{*}\left(S^{1}\right)$.

TheOrem 5. $D^{n}-L$ is a homotopy circle.

Proof. By the preceding proposition, $D^{n}-L$ has the same homotopy groups as a circle. But $D^{n}-L$ is homotopy equivalent to $C$, which is homeomorphic to a finite simplicial complex. Since the inclusion $i: S^{1} \rightarrow C$ of a meridian of $L$ induces the isomorphism $\pi_{1}\left(S^{1}\right) \rightarrow \pi_{1}(C)$, we can conclude that $i$ is a homotopy equivalence. Thus $C \sim_{\text {h.e. }} D^{n}-L$ is a homotopy circle.

It only remains to show that $L$ is non-trivial, which will follow once we show that the group $\pi_{1}(X)$ of the boundary knot $\partial L$ is not $\mathbb{Z}$.

Lemma 6. The group $G=\left\langle a, b \mid b^{2} a b a^{-1} b^{-1} a b^{-1} a^{-1}\right\rangle$ is not isomorphic to $\mathbb{Z}$.

Proof. This lemma can be proven in a variety of ways. The following elegant demonstration was shown to me by Andrew Casson.

We adjoin an extra generator $c$, which we immediately set equal to $a b a^{-1}$. Then

$$
\begin{aligned}
\left\langle a, b \mid b^{2} a b a^{-1} b^{-1} a b^{-1} a^{-1}\right\rangle & \cong\left\langle a, b, c \mid b^{2} a b a^{-1} b^{-1} a b^{-1} a^{-1}, c a b^{-1} a^{-1}\right\rangle \\
& \cong\left\langle a, b, c \mid b^{2} c b^{-1} c^{-1}, c a b^{-1} a^{-1}\right\rangle \\
& \cong \frac{\left\langle b, c \mid b^{2} c b^{-1} c^{-1}\right\rangle *\langle a\rangle}{\left\langle c a b^{-1} a^{-1}\right\rangle} .
\end{aligned}
$$

Written this way, $G$ has the form of an HNN extension of the BaumslagSolitar group $H=\left\langle b, c \mid b^{2} c b^{-1} c^{-1}\right\rangle$, which is isomorphic to the semidirect product $\mathbb{Z}[1 / 2] \rtimes \mathbb{Z}$. Thus $H$ is a non-abelian subgroup of $G$, which hence cannot be $\mathbb{Z}$.

Alternatively, to apply an unnecessarily large hammer, once $G$ is written as $\left\langle a, b, c \mid b^{2} a b a^{-1} b^{-1} a b^{-1} a^{-1}, c a b^{-1} a^{-1}\right\rangle$, it follows from [9] that $G$ is not even residually finite.

A third proof would utilize Whitehead's theorem on one-relator groups [12].

REMARK 7 . There is nothing exceptionally special about the group $G$ we have used in this construction, except that it turned out to be a fairly tractable example of a group with suitable properties. Any group possessing a two-generator, one-relator presentation with the properties employed above clearly would be sufficient. 


\section{G. Friedman}

\section{References}

[1] J. Boersema and E. J. Taylor, Knots in four dimensions and the fundamental group, http://www.rose-hulman.edu/mathjournal/2003/vol4-n2/paper2/v4n2-2pd.pdf.

[2] M. Freedman, The disk theorem for four-dimensional manifolds, in: Proc. Internat. Congress of Mathematicians (Warszawa, 1983), Vol. 1, PWN - Polish Scientific Publishers, Warszawa, 1984, 647-663.

[3] M. H. Freedman and F. Quinn, Topology of 4-manifolds, Princeton Univ. Press, Princeton, NJ, 1990.

[4] G. Friedman, Alexander polynomials of non-locally-flat knots, Indiana Univ. Math. J. 52 (2003), 1479-1578.

[5] J. F. P. Hudson, Piecewise Linear Topology, W. A. Benjamin, New York, 1969.

[6] J. Levine, Unkotting spheres in codimension two, Topology 4 (1965), 9-16.

[7] -, Polynomial invariants of knots of codimension two, Ann. of Math. 84 (1966), $537-554$.

[8] -, An algebraic classification of some knots of codimension two, Comment. Math. Helv. 45 (1970), 185-198.

[9] E. Raptis, O. Talelli and D. Varsos, On the Hopficity of certain HNN-extensions with base a Baumslag-Solitar group, Algebra Colloq. 9 (2002), 39-48.

[10] D. Rolfsen, Knots and Links, Publish or Perish, Berkeley, CA, 1976.

[11] J. Stallings, On topologically unknotted spheres, Ann. of Math. (2) 77 (1963), 490-503.

[12] J. H. C. Whitehead, On equivalent sets of elements in a free group, ibid. 37 (1936), 782-800.

Department of Mathematics

Vanderbilt University

1326 Stevenson Center

Nashville, TN 37240, U.S.A.

E-mail: greg.friedman@vanderbilt.edu

Received 7 July 2005;

in revised form 18 May 2006 